\title{
Evaluation of BAL versus sputum specimen for the diagnosis of pulmonary tuberculosis in sputum AFB smear negative patients- retrospective study at PIMSR, Gujarat
}

\author{
Vaidehi. J. Mehta ${ }^{1}$, Mohmed Soeb Jankhwala,", Anant Marathe ${ }^{3}$ \\ 1,2 Assistant Professor, ${ }^{3}$ Professor \& HOD, Dept. of Microbiology, Parul Institute of Medical Sciences \& Research, Waghodia, \\ Gujarat, India
}

*Corresponding Author:

Email: shoaibdoc4@gmail.com

\begin{abstract}
Aims and Objective: Early diagnosis of smear negative tuberculosis is a significant challenge in control of tuberculosis. The present retrospective study was done on sputum and BAL samples of the same patients for better diagnosis of pulmonary tuberculosis.

Material and Methods: A total of 1000 (700 sputum and 300 BAL) specimens were processed to rule out tuberculosis in suspected cases during the period of August 2015 to March 2018. The result of sputum as well as BAL specimens from 300 same patients were examined for ZN staining and GeneXpert tests. The samples of sputum and BAL were processed as per the standard guidelines.

Results: Total of 1000 specimens (700 sputum \& 300 BAL) were considered for the results. Out of 1000 specimens 300 patients had submitted both sputum as well as BAL specimens. These 300 specimens were analyzed for comparative results.

Out of 300 common samples, 109 (36.3\%) sputum and $136(45 \%)$ BAL samples are positive by ZN stain. Whereas, Out of 300 common samples, $149(49.6 \%)$ sputum and $170(56.6 \%)$ BAL samples are positive by GeneXpert method. Out of 149 common specimens that were positive by GeneXpert, 5(3.3\%) were found to be Rifampicin resistant.

Conclusion: Incidence of MDR TB is 3.3\% in our hospital. BAL was found to be superior specimen to sputum for the diagnosis of ZN smears negative pulmonary TB. Sensitivity and specificity of GeneXpert is higher than AFB smear microscopy.
\end{abstract}

Keywords: Sputum, BAL, GeneXpert, MDR-TB (Multidrug Resistance Tuberculosis).

\section{Introduction}

The diagnosis of tuberculosis is a challenge in developing countries. ${ }^{1}$ For decades sputum smear examination was routinely used for the diagnosis of pulmonary tuberculosis. Many patients fail to produce sputum. Among the patients who are able to expectorate sputum, a significant number of times the AFB staining fails to detect AFB even in the patients who have radiological evidence of TB in chest X-ray. ${ }^{2}$

A particular challenge for clinicians concerns the rising incidence of human immunodeficiency virus (HIV) related TB, with an associated increase in smear negative TB. Smear negative HIV related TB has an increased mortality compared to smear positive disease and this may in part be related to delays in diagnosis and initiation of treatment. ${ }^{2}$

Sputum smear microscopy to detect acid-fast bacilli (AFB) is a rapid, inexpensive, and highly specific tool for identifying persons with active pulmonary tuberculosis but it has low sensitivity. ${ }^{2}$ On the other side, nucleic acid amplification techniques (GeneXpert) due to its rapidity and sensitivity not only help in early diagnosis and management of tuberculosis especially in patients with high clinical suspicion like immunocompromised patients, history of contact with active tuberculosis patient etc, but also curtail the transmission of the disease. ${ }^{3}$

The current study was undertaken to evaluate the relative positivity by AFB smear and GeneXpert study of bronchoalveolar lavage (BAL) samples for the diagnosis of $M$. tuberculosis in sputum AFB smearnegative patients.

\section{Material and Methods}

The present study is carried out in the Department of Microbiology in a tertiary care hospital in Gujarat. Total 1000 patients were studied during the period from August 2015 to March 2018.

Inclusion Criteria: Patients with strong suspicion of pulmonary TB viz. fever with cough for more than 2 weeks, history of hemoptysis, loss of weight and anorexia are included.

Collection and Transport of Specimen: Patients suspected of pulmonary TB were initially investigated for sputum acid-fast smear at least on two consecutive days. Patients with a repeated sputum AFB negative with strong clinico-radiological suspicion of pulmonary tuberculosis were then counseled, consented and subjected to fiber-optic bronchoscopy for collection of BAL.

Procedure: Bronchoscopy procedure was performed with Olympus fiber optic microscope and its accessories for the collection of BAL fluid.

Each sputum or BAL specimen was processed for decontamination using $4 \% \mathrm{NaOH}$ and then centrifuged at 3000 revolutions per minute for 20 minutes after discarding the supernatant, sediments were neutralized with N/10 HCL. Sediments were used to prepare 
smears for $\mathrm{ZN}$ staining and part of it was used to process for PCR by GeneXpert. ${ }^{2}$

Laboratory Methods: Both sputum as well as BAL samples were processed for AFB smear preparation and GeneXpert assay. GeneXpert testing was performed according to the manufacturer's instructions. ${ }^{4}$

\section{Results}

Total of 1000 specimens (700 sputum \& 300 BAL) were considered for the results. Out of 1000 specimens 300 patients had submitted both sputum as well as BAL specimens. These 300 specimens were analyzed for comparative results.

Out of 300 common samples, 109 (36.3\%) sputum and $136(45 \%)$ BAL samples are positive by $\mathrm{ZN}$ stain. Whereas, out of 300 common samples, 149 (49.6\%) sputum and $170(56.6 \%)$ BAL samples are positive by GeneXpert method as shown in Chart 1.

\section{Chart: 1 Comparison of sputum vs BAL in same patients}



Out of 149 common specimens that were positive by GeneXpert, 5(3.3\%) were found to be Rifampicin resistant as shown in Chart 2.

Chart 2: Shows the prevalence of MDR TB

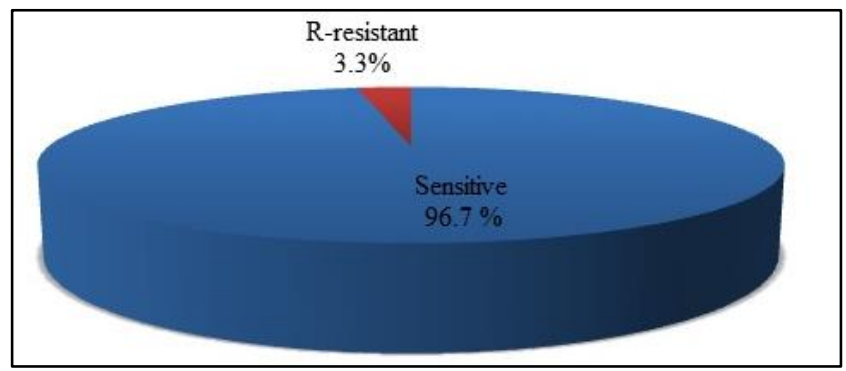

\section{Analysis}

Statistical analysis of the data was done to find out Sensitivity, specificity, PPV and NPV. The sensitivity, specificity, PPV and NPV for the diagnosis of pulmonary tuberculosis was calculated for AFB smear microscopy and the GeneXpert, using GeneXpert as gold standard. By taking GeneXpert as reference, samples that were positive and negative in GeneXpert were considered true positive and true negative.

Table 1: Shows the comparative results of BAL samples for ZN stain and GeneXpert

\begin{tabular}{|c|c|c|c|c|}
\hline BAL & \multicolumn{2}{|c|}{ ZN Stain } & \multicolumn{2}{c|}{ GeneXpert } \\
\hline & Positive & Negative & Positive & Negative \\
\hline Total 300 & 136 & 164 & 170 & 130 \\
\hline
\end{tabular}

Table 2: Shows the comparative results of sputum samples for $\mathrm{ZN}$ stain and GeneXpert

\begin{tabular}{|c|c|c|c|c|}
\hline Sputum & \multicolumn{2}{|c|}{ ZN Stain } & \multicolumn{2}{c|}{ GeneXpert } \\
\hline & Positive & Negative & Positive & Negative \\
\hline Total 300 & 109 & 191 & 149 & 151 \\
\hline
\end{tabular}

In the study we found higher sensitivity of GeneXpert test as there were 170 samples positive by GeneXpert while 136 were positive by $\mathrm{ZN}$ staining in case of BAL specimen. In case sputum specimen 149 were positive by GeneXpert while $\mathrm{ZN}$ staining gave 109 positive results. There were some samples which were positive by $\mathrm{ZN}$ staining but negative by GeneXpert. In present study we have found 1 in case of sputum while 2 in case of BAL were found positive by
$\mathrm{ZN}$ staining. This can be explained by the fact that GeneXpert could detect only mycobacterium tuberculosis while $\mathrm{ZN}$ detects all AFBs.

\section{Discussion}

The diagnosis of pulmonary TB in patients with high index of suspicion but sputum smear negative for AFB becomes challenging. ${ }^{6}$ Sputum AFB although 
widely used and cheapest method has variable diagnostic yield. ${ }^{1}$

In the present study we have evaluated the comparative diagnostic yield of BAL samples over sputum samples for the diagnosis of pulmonary tuberculosis.

ZN stain is a very rapid, cost effective test but it has some limitations. GeneXpert is a molecular diagnostic assay that can be performed with minimal training. The results are available within 2 hours, much earlier than the culture which usually takes days to come positive. ${ }^{3,5,6}$

Numbers of studies have demonstrated the utility of GeneXpert from BAL samples in diagnosis of smear negative pulmonary tuberculosis. In our study, overall sensitivity, specificity, PPV and NPV of GeneXpert from BAL samples are 99\%, 100\%, 100\% and 99\% respectively that is comparable with other studies. ${ }^{8-12}$

Numbers of studies have demonstrated the utility $\mathrm{ZN}$ stain from BAL samples in diagnosis of smear negative pulmonary tuberculosis. ${ }^{8-12}$ In our study, overall sensitivity, specificity, PPV and NPV of ZN stain from BAL samples are $80 \%, 80 \%, 98 \%$ and $84 \%$ respectively that is comparable with other studies. ${ }^{8-12}$

Sensitivity, specificity, PPV and NPV of GeneXpert in sputum assay in our study is $98 \% 100 \%$, $100 \%$ and $98 \%$ that is line with the study of Sharma et al. ${ }^{13}$ (96.9\% and $99.8 \%$ )

In comparison with GeneXpert, as gold standard, sensitivity, specificity, PPV and NPV for ZN smear microscopy for sputum samples is $73 \%, 99 \%, 99 \%$ and $82 \%$. Monika Agrawal ${ }^{3}$ also reported the same $72.7 \%$, $100 \%, 100 \%$ and $76.9 \%$.

GeneXpert assay had an overall sensitivity of $99 \%$ for BAL and $98 \%$ for sputum samples for PTB, which is superior to that of smear microscopy $80 \%$ and $73 \%$ for BAL and sputum respectively which is also correlated well with other studies. ${ }^{8-12}$

\section{Conclusion}

Incidence of MDR TB is 3.3\% in our hospital. BAL was found to be superior specimen to sputum for the diagnosis of $\mathrm{ZN}$ smear negative pulmonary TB. Sensitivity and specificity of GeneXpert is higher than AFB smear microscopy. GeneXpert in smear positive pulmonary samples also, to know the infection with MTB and its Rifampicin susceptibility pattern. According to the study BAL specimen has advantage over sputum specimen in the diagnosis of pulmonary tuberculosis. Detection of Rifampicin resistance by GeneXpert is extremely useful as there is rise in cases of MDR tuberculosis. Wide application of GeneXpert test will help rapid diagnosis, early treatment and better control of tuberculosis in India. and post bronchoscopy sputum and bronchoalveolar lavage in HIV infected patients with atypical chest X-rays in Ethiopia. Ann Thorac Med 2007 Oct-Dec;2(4):154157.

2. George $\mathrm{P}$, Mehta $\mathrm{M}$ et al. Post-bronchoscopy sputum: Improving the diagnostic yield in smear negative pulmonary TB. J Respiratory Medicine 2011;1(1):172631.

3. Agrawal M, Bajaj A et al. Comparative study of GeneXpert with ZN stain and culture in samples of suspected pulmonary Tubeculosis. J of Clin and Dia research 2016 May;10(5):9-11.

4. XpertMTB RIF kit insert http://www.cepheid.com/manageddownloads/xpertmtbrif-english-package-insert-301-1404-rev-b-february2015.pdf

5. Iyer V, Joshi A Y, Boyce T G et al. Bronchoscopy in suspected pulmonary TB with negative induced sputum smear and MTD Gen-probe testing. J.rmed. 2011.March;105,1084-90.

6. Forbes BA, Sahm DF, Weissfeld AS. Bailey and Scott's Diagnostic Microbiology, $12^{\text {th }}$ edition. The C.V Mosby Co. St.Louis. 2007, chapter 45 Pg: 478-509.

7. World Health Organization: Automated real-time nucleic acid amplification technology for rapid and simultaneous detection of tuberculosis and rifampicin resistance: Xpert $\mathrm{mtb} / \mathrm{rif}$ system. Policy statement 2011. http://whqlibdoc.who. int/publications/2011/9789241501545_eng.pdf

8. Barnard DA, Irusen EM, Bruwer JW, Plekker D, Whitelaw AC, Deetlefs JD, et al. Koegelenberg The utility of Xpert MTB/RIF performed on bronchial washings obtained in patients with suspected pulmonary tuberculosis in a high prevalence setting. BMC Pulm Med. 2015;15:103

9. Bhatia R, Ichhpujani RL. Essential of medical microbiology. 4th ed. Jaypee;2008.

10. Pierre Le Palud P, Cattoir V, Malbruny B, Magnier R, Campbell K, Oulkhouir Y, et al. Retrospective observational study of diagnostic accuracy of the Xpert@MTB/RIF assay on fiberoptic bronchoscopy sampling for early diagnosis of smearnegative or sputumscarce patients with suspected tuberculosis. BMC Pulm Med.2014;14 (1):137.

11. Lee HY, Seong MW, Park SS, Hwang SS, Lee J, Park YS, et al. Diagnostic accuracy of Xpert®MTB/RIF on bronchoscopy specimens in patients with suspected pulmonary tuberculosis. Int J Tuberc Lung Dis. 2013;17(7):917-21.

12. Sharma SK, Kohli M, Yadav RN, Chaubey J, Bhasin D, Sreenivas V, et al. Evaluating the diagnostic accuracy of xpert mtb/rif assay in pulmonary tuberculosis. www.journals.plos.org/plosone/article?id=10.1371/journa 1.pone.0141011.

13. Khalil KF, Butt T. Diagnostic yield of bronchoalveolar lavage gene xpert in smear- negative and sputum-scarce pulmonary tuberculosis. Journal of the College of Physicians and Surgeons Pakistan. 2015;25(2):115-18.

\section{References}

1. Getachew A, Haimot $\mathrm{G}$ et al. Comparison of acid fast stain and culture for Mycobacterium tuberculosis in pre 